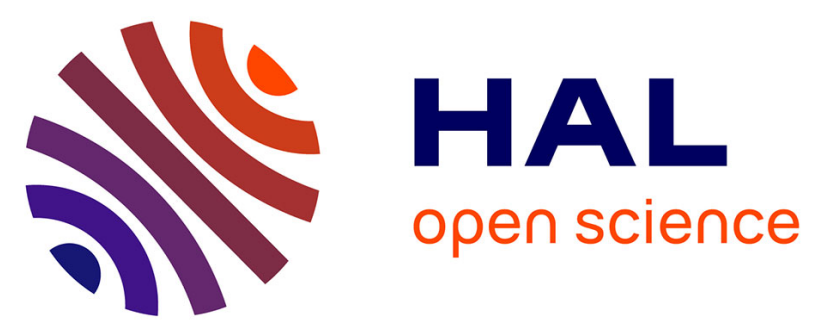

\title{
Monte Carlo simulation of confined fluids of polarizable particles:an efficient iterative treatment of the local field in slab geometry using Ewald summation
}

\author{
Charles Brunet, Jean Guillaume Malherbe, Saïd Amokrane
}

\section{- To cite this version:}

Charles Brunet, Jean Guillaume Malherbe, Saïd Amokrane. Monte Carlo simulation of confined fluids of polarizable particles:an efficient iterative treatment of the local field in slab geometry using Ewald summation. Molecular Physics, 2010, 108 (13), pp.1773-1781. 10.1080/00268976.2010.490794 . hal00608031

\section{HAL Id: hal-00608031 \\ https://hal.science/hal-00608031}

Submitted on 12 Jul 2011

HAL is a multi-disciplinary open access archive for the deposit and dissemination of scientific research documents, whether they are published or not. The documents may come from teaching and research institutions in France or abroad, or from public or private research centers.
L'archive ouverte pluridisciplinaire HAL, est destinée au dépôt et à la diffusion de documents scientifiques de niveau recherche, publiés ou non, émanant des établissements d'enseignement et de recherche français ou étrangers, des laboratoires publics ou privés. 


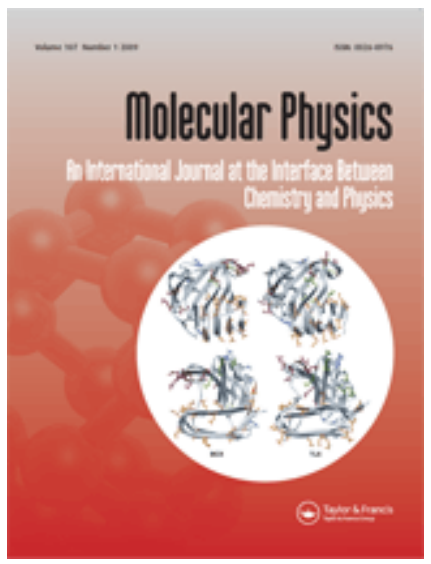

\section{Monte Carlo simulation of confined fluids of polarizable particles:an efficient iterative treatment of the local field in slab geometry using Ewald summation}

\begin{tabular}{|c|c|}
\hline Journal: & Molecular Physics \\
\hline Manuscript ID: & TMPH-2009-0430.R2 \\
\hline Manuscript Type: & Full Paper \\
\hline $\begin{array}{r}\text { Date Submitted by the } \\
\text { Author: }\end{array}$ & 13-Apr-2010 \\
\hline Complete List of Authors: & $\begin{array}{l}\text { Brunet, Charles; Université paris Est (Créteil) } \\
\text { Malherbe, Jean Guillaume; PLMC, Faculté des sciences \& tech } \\
\text { Amokrane, Saïd; PLMC, Faculté des Sciences et Technologie }\end{array}$ \\
\hline Keywords: & dipolar interaction, polarizability, confined fluid, simulation \\
\hline \multicolumn{2}{|c|}{$\begin{array}{l}\text { Note: The following files were submitted by the author for peer review, but cannot be converted } \\
\text { to PDF. You must view these files (e.g. movies) online. }\end{array}$} \\
\hline TMPH-2009-0430.R2.zip & \\
\hline
\end{tabular}

\section{ScholarONE \\ Manuscript Central}




\title{
Monte Carlo simulation of confined fluids of polarizable particles: an efficient iterative treatment of the local field in slab geometry using Ewald summation
}

\author{
C. Brunet, J. G. Malherbe and S. Amokrane \\ Physique des Liquides et Milieux Complexes, Faculté des Sciences et de \\ Technologie,Université Paris Est, 61 av. du Général de Gaulle, 94010 Créteil Cedex, \\ France \\ (Received 00 Month 200x; in final form 00 Month 200x)
}

\begin{abstract}
We propose a method for treating in Monte Carlo simulations the problem of the induced dipoles for polarizable particles fluids confined in slab geometry and subject to an external field. In order to compute the local field in a reasonable time, a partial update of the induced dipole moments is performed by introducing a cut-off distance, as in bulk systems. This strategy is then combined with a slab adapted 3D-Ewald summation for treating the longrange interactions between the induced dipoles. The method is illustrated by simulations of confined binary mixtures in the canonical and grand canonical ensembles.
\end{abstract}

\section{Introduction}

The study of dipolar fluids by simulation has become rather common since the mid-eighties (see for example [1-4]). However, relatively few studies dealt with the case of polarizable particles. One reason for this is a practical difficulty. Indeed, when the dipole moments are induced by the applied field, the polarization in the whole system must be updated after each particle move, in the Monte Carlo (MC) method (see for example [5]). The result is a critical slowing down of the simulation, in comparison with the situation of permanent dipoles. Special MC techniques were recently proposed to overcome this problem, such as the one proposed by Moučka et al. [6] that treats the polarization by multi-particle force-biased moves. Shortly before, Předota et al. [7] proposed an approximate method, the pair approximation for polarization interactions (PAPI) to treat the polarization by introducing a cutoff distance beyond which the local field should not not be affected significantly by a local MC move. While both methods result in a drastic increase in the simulation efficiency, they share the same ingredient to handle long range interactions, namely the reaction field method. The latter is not adapted for the slab geometry which occurs frequently in surface and interfacial systems because of its mean-field nature. As a result, one usually prefers to use in this case the Ewald summation method (see [8] and refs therein).

The specific situation of induced dipoles in slab geometry, raises thus two different problems whose combination has, to our knowledge, not been considered before. The first one is the treatment of the long-range dipolar interaction, for a given distribution of the dipole moments. The second one is the time needed to compute the induced dipoles. We thus show in this paper that it is possible to combine the slab adapted 3D-Ewald summation with the appropriate treatment of the local field to efficiently treat induced dipoles confined in slab geometry.

The practical motivation of this study is the development of experiments on con-

Molecular Physics

ISSN 00268976 print/ISSN 13623028 online (c) 200x Taylor \& Francis

http://www.tandf.co.uk/journals

DOI: $10.1080 / 0026897 \mathrm{YYXxxxxxxx}$

URL: http://mc.manuscriptcentral.com/tandf/tmph 
fined colloidal particles. In this domain, permanent dipoles are usually found in ferromagnetic colloids and ferroelectric nematic ones (spherical ferroelectric particles that can be dispersed in solution have been elaborated more recently [9]). Polarizable colloidal particles are on the other hand rather common, since polarizability of the materials forming the colloids, say the electronic one, is always present to some extent. Examples are polymethylmethacrylate (PMMA) or polystyrene Latex particles. Polarizability is also quite frequent in core-shell nanoparticles [10]). Furthermore, the consideration of an external field in confined geometry gives additional flexibility in the control of the behavior of a confined dipolar fluid [11-13].

Studying polarizable models in confinement is thus of much practical interest, besides the general question of the treatment of non-additive interactions [6]. We hence show in this work that the local field can be computed efficiently also in slab geometry. To this end, this paper is organized as follows: we first present the model of confined polarizable fluids that we will study. We then propose the method to treat the polarization for slab geometry by the Ewald summation. The efficiency of the proposed method is shown by simulating mixtures of polarizable and non-polarizable particles in the canonical and grand canonical ensembles. This will extend our previous results $[14,15]$ relative to permanent dipoles to the case of induced ones.

\section{Polarizability and local field}

\subsection{Potential Energy for a model with polarizability}

We consider polarizable particles confined between two parallel walls. In this model, the polarizability is accounted for by a point dipole $\boldsymbol{\mu}_{\mathrm{i}}$, taken proportional to the local electric field $\boldsymbol{E}^{\text {loc }}$ at the position of the center of mass $\boldsymbol{r}_{i}$ of particle $i$ :

$$
\boldsymbol{\mu}_{\mathrm{i}}=\alpha_{p} \boldsymbol{E}^{\mathrm{loc}}\left(\boldsymbol{r}_{i}\right)
$$

$\alpha_{p}$ is here taken as a scalar for simplicity. The local field involves two contributions :

$$
\boldsymbol{E}^{\mathrm{loc}}\left(\boldsymbol{r}_{i}\right)=\boldsymbol{E}^{\mathrm{ext}}\left(\boldsymbol{r}_{i}\right)+\boldsymbol{E}^{\mu}\left(\boldsymbol{r}_{i}\right)
$$

where $\boldsymbol{E}^{\text {ext }}$ the external field and $\boldsymbol{E}^{\mu}$ is the field due to the other induced dipoles :

$$
\boldsymbol{E}^{\mu}\left(\boldsymbol{r}_{i}\right)=\sum_{j \neq i} \boldsymbol{T}_{i j} \cdot \boldsymbol{\mu}_{\mathrm{j}}
$$

with

$$
\boldsymbol{T}_{i j}=\frac{1}{r_{i j}^{3}}\left(\frac{3 \boldsymbol{r}_{i j} \boldsymbol{r}_{i j}}{r_{i j}^{2}}-\boldsymbol{I}\right)
$$

where $\boldsymbol{I}$ is the identity matrix. The induced dipoles are then given by the system of equations:

$$
\boldsymbol{\mu}_{\mathrm{i}}=\alpha_{p}\left(\boldsymbol{E}^{\mathrm{ext}}+\sum_{\substack{j \\ j \neq i}} \boldsymbol{T}_{i j} \cdot \boldsymbol{\mu}_{\mathrm{j}}\right)
$$


Since the direct solution of eq. (5) is impractical [17], it is more convenient to compute the local field by an iterative scheme. Indeed, the local field $\boldsymbol{E}^{\text {loc }}\left(\boldsymbol{r}_{i}\right)$ inducing the dipole moment $\boldsymbol{\mu}_{\mathrm{i}}$ at the position $\boldsymbol{r}_{i}$ depends on all the dipole moments for the actual spatial configuration of the system $\boldsymbol{E}^{\mathrm{loc}}\left(\boldsymbol{r}_{i}\right)=\boldsymbol{E}^{\mathrm{loc}}\left(\boldsymbol{r}_{i} ; \boldsymbol{\mu}_{\mathrm{j}}\left(\left\{\boldsymbol{r}_{k}\right\}\right)\right)$.

Now, the difference with permanent dipoles is that one needs to evaluate the local field before computing the energy. A convenient expression of the local field can be derived starting from the potential energy for polarizable particles in an external field $U$ written (see for example $[16,17]$ ) in the form :

$$
U=U^{\mu \mu}+U^{\mathrm{ext}}+U^{\mathrm{pol}}
$$

The first two terms in eq. (6) are the dipole-dipole and dipole-external field energies, as if the dipoles were permanent :

$$
\begin{gathered}
U^{\mu \mu}=-\frac{1}{2} \sum_{i \neq j} \boldsymbol{\mu}_{\mathrm{i}} \cdot \boldsymbol{T}_{i j} \cdot \boldsymbol{\mu}_{\mathrm{j}} \\
U^{\mathrm{ext}}=-\sum_{i} \boldsymbol{\mu}_{\boldsymbol{i}} \cdot \boldsymbol{E}^{\mathrm{ext}}
\end{gathered}
$$

and $U^{\text {pol }}$ is the polarization energy [16] or the work done against the internal forces, to form the dipoles in the local field :

$$
U^{\mathrm{pol}}=\frac{1}{2} \sum_{i} \boldsymbol{\mu}_{\mathrm{i}} \cdot \boldsymbol{E}^{\mathrm{loc}}\left(\boldsymbol{r}_{i}\right)
$$

When the relation eq. (1) between the induced dipoles and the local field is used, this expression becomes

$$
U^{\mathrm{pol}}=\frac{1}{2 \alpha_{p}} \sum_{i} \boldsymbol{\mu}_{\mathrm{i}}^{2}
$$

Since this additional term involves only the local field any technique to handle long range interactions for permanent dipoles will also work for polarizable particles. The local field can then be obtained as

$$
\boldsymbol{E}^{\mathrm{loc}}=-\frac{\partial\left(U-U^{\mathrm{pol}}\right)}{\partial \boldsymbol{\mu}_{\mathrm{i}}}
$$

Once the induced dipoles are known, the total energy in eq. (6) can be conveniently evaluated with the help of eq. (7), eq. (8) and eq. (9) as:

$$
U=-\frac{1}{2} \sum_{i} \boldsymbol{\mu}_{\mathrm{i}} \cdot \boldsymbol{E}^{\mathrm{ext}}
$$

The problem of the treatment of the periodic boundary conditions is however still present in the evaluation of the induced dipole moments $\boldsymbol{\mu}_{\mathrm{i}}$. This is discussed in the next section. 


\subsection{Local field in the Ewald-Summation}

In slab geometry, adapted 3D-Ewald summation techniques [18-21] are usually used and the local field is derived from the Ewald energy. In the Ewald sum in which the dipole-dipole term is written as $U^{\mu \mu}=U^{(r)}+U^{(k)}+U^{(s)}$, each term in the potential energy gives a contribution to the local field computed from eq. (11) (see for example $[8,22]$ ). The short-range contribution $U^{(r)}$ may be written as :

$$
U^{(r)}=-\frac{1}{2} \sum_{i \neq j} \mu_{i, s}\left(T^{(r)}\right)_{i j}^{s t} \mu_{j, t}
$$

with

$$
\left(T^{(r)}\right)_{i j}^{s t}=C\left(r_{i j}, \alpha\right) r_{i j}^{s} r_{i j}^{t}-\delta^{s t} B\left(r_{i j}, \alpha\right)
$$

where $s$ and $t$ stand for the space coordinates $\{x, y, z\}$ (Einstein's convention for repeated indexes is used); $\delta$ is the Kronecker symbol and the functions $\mathrm{B}$ and $\mathrm{C}$ are defined by :

$$
\begin{gathered}
\mathrm{B}(r, \alpha)=\operatorname{erfc}(\alpha r) / r^{3}+\left(2 \alpha / \pi^{1 / 2}\right) \exp \left(-\alpha^{2} r^{2}\right) / r^{2} \\
\mathrm{C}(r, \alpha)=3 \operatorname{erfc}(\alpha r) / r^{5}+\left(2 \alpha / \pi^{1 / 2}\right)\left(2 \alpha^{2}+3 / r^{2}\right) \exp \left(-\alpha^{2} r^{2}\right) / r^{2}
\end{gathered}
$$

The corresponding contribution to the local field reads:

$$
E_{i, s}^{(\mathrm{r})}=\sum_{\substack{j \\ j \neq i}}\left(T^{(r)}\right)_{i j}^{s t} \mu_{j, t}
$$

The long-range contribution to the energy is given by :

$$
U^{(k)}=-\frac{1}{2} \sum_{i, j} \mu_{i, s}\left(T^{(k)}\right)_{i j}^{s t} \mu_{j, t}
$$

with

$$
\left(T^{(k)}\right)_{i j}^{s t}=-\frac{1}{L^{3}} \sum_{k \neq 0} \frac{4 \pi}{k^{2}} \exp \left(-\frac{k^{2}}{4 \alpha^{2}}\right) k^{s} k^{t} \exp \left[i \boldsymbol{k} \cdot\left(\boldsymbol{r}_{i}-\boldsymbol{r}_{j}\right)\right]
$$

and the corresponding long-range contribution to the field in the $s$-direction is :

$$
E_{i, s}^{(\mathrm{k})}=\sum_{j}\left(T^{(k)}\right)_{i j}^{s t} \mu_{j, t}
$$

The self-energy term that eliminates unwanted self-interactions in the central box is :

$$
U^{(\mathrm{s})}=-\frac{2 \alpha^{3}}{3 \sqrt{\pi}} \sum_{i} \boldsymbol{\mu}_{\mathrm{i}} \cdot \boldsymbol{\mu}_{\mathrm{i}}
$$


It contributes to the local field by :

$$
E_{i, s}^{(\mathrm{s})}=\frac{4 \alpha^{3}}{3 \sqrt{\pi}} \mu_{i, s}
$$

In the standard 3D-Ewald-scheme, an additional term must be added that depends on the dielectric medium surrounding the replicated boxes. For slab geometry, one actually incorporates a vacuum region between the central slab and its replicas as proposed by Yeh and Berkowitz [18]. This introduces a correction to the energy and thus to the field given by :

$$
\boldsymbol{E}^{(c)}=4 \pi P_{z} \boldsymbol{u}_{z}
$$

where $\boldsymbol{P}$ is the total polarization in the slab.

As a result, in Slab geometry, the component in the $s$-direction of the local field acting on the particle $i$ is :

$$
E_{i, s}^{\mathrm{local}}=E_{i, s}^{\mathrm{ext}}+E_{i, s}^{(\mathrm{r})}+E_{i, s}^{(\mathrm{k})}+E_{i, s}^{(\mathrm{s})}+\delta^{s z} E_{s}^{(c)}
$$

As a verification, one can check that for permanent dipoles, the potential energy obtained with this local field as $U=U^{\text {ext }}-\frac{1}{2} \sum_{i} \boldsymbol{\mu}_{\mathrm{i}} \cdot\left(\boldsymbol{E}^{\text {loc }}\left(\boldsymbol{r}_{\boldsymbol{i}}\right)-\boldsymbol{E}^{\text {ext }}\right)$ is consistent with the standard Ewald Energy route $U^{e x t}+U^{(r)}+U^{(k)}+U^{(s)}$. For polarizable particles the energy is obtained from eq. (12) as recalled above. One known problem with the original method is that the vacuum region can be quite large. To reduce the gap width the so-called electrostatic layer correction (ELC) has been introduced, initially for point charges [20] and later for dipoles [21]. Furthermore the ELC method and its variants allow an a priori control of the errors, this being important to determine the optimum conditions for using the method (see for example [23]). Here, however, our purpose was to investigate the question of the local field, which is a distinguishing feature in comparison with permanent dipoles. Of course, all the improvements made in the latter case can and should be implemented, in a second stage to study induced ones.

\section{Iteration process for the local field with polarizable particles}

\subsection{The full update process}

We now turn to the implementation of these considerations in Monte Carlo simulation. For polarizable particles with point dipoles, only the positions $\boldsymbol{r}_{i}$ need to be sampled. But even a local displacement of one particle yields a change of the local field acting on all the other particles. After a move of particle $i$ from $\boldsymbol{r}_{i}^{\text {old }}$ to $\boldsymbol{r}_{i}^{\text {new }}$, the new induced dipole moment is $\boldsymbol{\mu}_{\mathrm{i}}^{\text {new }}=\alpha_{p} \boldsymbol{E}_{\text {loc }}^{\text {new }}\left(\boldsymbol{r}_{i}^{\text {new }}\right)$. This modifies the local field acting on the other particles and hence one has $\boldsymbol{\mu}_{\mathrm{j}}^{\text {new }}=\alpha_{p} \boldsymbol{E}_{\mathrm{loc}}^{\text {new }}\left(\boldsymbol{r}_{j}\right)$. As a result, after each local change (particle move, or more generally insertion, etc.) one needs to update all the dipole moments in an iterative way to compute the local field. In an algorithm with full update, all the induced dipole moments are sequentially updated according to $\boldsymbol{\mu}_{\mathrm{i}}^{(\mathrm{n})}=\alpha_{p} \boldsymbol{E}_{\text {loc }}\left(\left\{\boldsymbol{\mu}_{\mathrm{j}<\mathrm{i}}^{(\mathrm{n})}\right\},\left\{\boldsymbol{\mu}_{\mathrm{j} \geq \mathrm{i}}^{(\mathrm{n}-1)}\right\}\right)$ until one observes for all dipoles :

$$
\frac{\left|\Delta \boldsymbol{\mu}_{i}\right|}{\left|\boldsymbol{\mu}_{i}\right|}<\epsilon
$$


where $\left|\Delta \boldsymbol{\mu}_{i}\right|$ is the magnitude of the variation of the dipole moment between iterations $(n-1)$ and $(n)$. With $\epsilon=10^{-4}$, we checked that the energy is affected only by a term of the order of the machine single-precision error. This criterion is satisfied after approximately 5 iterations. The time required to evaluate the change in energy by this method after $N$ particle position updates is then much larger than in simulations with permanent dipoles. This full update algorithm would require a considerable amount of computer time, for example in order to obtain very accurate results in a specific situation (see for example the benchmark result on water of Jedlovszky et al. [5]). Faster - albeit probably less accurate - variants are thus needed to explore the parameters space of a generic model in a reasonable time. One possibility is considered below.

\section{$3.2 \quad$ Update process with a Cut-off}

Following this objective, the pair approximation for polarization interaction was proposed by Předota and coworkers [7] in the context of bulk polarizable fluids. The idea of this method is to update only the dipole moments of the particles that are within a given distance $R_{\text {cut }}$ from a displaced particle (see figure 2 in ref. [7] for implementation details). Only interactions between pairs are taken into account during the update process. Because of the long-range nature of the dipolar interaction it is clear that introducing cutoff distances can give only an approximation of the true local field. However, when the cut-off distance is taken large enough and the local change not too important, the thermodynamics should remain correct in this approximate treatment.

In slab geometry, one cannot use the reaction field method both because of the loss of the spherical symmetry and the problem of the meaning of the dielectric constant at high confinement. Since the PAPI method uses the reaction field, it must be adapted to deal with such a geometry. As depicted in Fig. 1 the zone inside which convergence of the local field will be made is a cylinder of height $H$ and radius $R_{\text {cut }}$ around the displaced particle. During the iteration process with the cut-off distance, only the dipole moments $\boldsymbol{\mu}^{\text {in }}$ inside the cylinder will be updated. Since the others, $\boldsymbol{\mu}^{\text {out }}$, are kept unchanged, their contribution to the local field acting on the particles inside the cylinder is fixed. The iteration process to compute the new dipole moments $\boldsymbol{\mu}^{\text {in }}$ is as follows :

- A random trial move $d \boldsymbol{r}$ for particle $i_{\mathrm{mv}}$ is chosen:

$$
\boldsymbol{r}_{i_{\mathrm{mv}}}^{\text {new }}=\boldsymbol{r}_{i_{\mathrm{mv}}}^{\text {old }}+d \boldsymbol{r}
$$

- The ensemble $\mathcal{D}_{\text {cut }}$ of the particles inside the cylinder is selected :

$$
\mathcal{D}_{\text {cut }}=\left\{i ; \sqrt{\left(r_{i, x}-r_{i_{\mathrm{mv}}, x}\right)^{2}+\left(r_{i, y}-r_{i_{\mathrm{mv}}, y}\right)^{2}} \leq R_{\mathrm{cut}}\right\}
$$

- The contribution of the out-dipoles to the local field acting on these particles is evaluated from the non-updated values $\boldsymbol{\mu}^{\text {out }}\left(j \notin \mathcal{D}_{\text {cut }}\right)$ :

$$
E_{i, s}^{\text {out }}=\sum_{j \notin \mathcal{D}_{\text {cut }}}\left(T^{(r)}\right)_{i j}^{s t} \mu_{j, t}+\sum_{j \notin \mathcal{D}_{\text {cut }}}\left(T^{(k)}\right)_{i j}^{s t} \mu_{j, t}+\delta^{s z} 4 \pi \sum_{j \notin \mathcal{D}_{\text {cut }}} \mu_{j, z}
$$

In practice, $E_{i, s}^{\text {out }}$ is more efficiently computed by subtracting the contribution of the actual dipoles located inside the cylinder from the stored values of the local fields computed during the previous Monte Carlo step (this is of course 
not possible for the displaced particle itself).

- The dipoles moments inside the cylinder $\boldsymbol{\mu}_{\mathrm{i}}^{\text {in }}\left(i \in \mathcal{D}_{\text {cut }}\right)$ are next modified :

$$
\boldsymbol{\mu}_{\mathrm{i}}^{\text {in }}=\alpha_{p} \boldsymbol{E}_{i}^{\mathrm{loc}}
$$

with

$$
\begin{aligned}
E_{i, s}^{\mathrm{loc}}= & E_{s}^{\mathrm{ext}}+E_{i, s}^{\mathrm{out}}+\sum_{\substack{j \in \mathcal{D}_{\text {cut }} \\
j \neq i}}\left(T^{(r)}\right)_{i j}^{s t} \mu_{j, t}+\sum_{j \in \mathcal{D}_{\text {cut }}}\left(T^{(k)}\right)_{i j}^{s t} \mu_{j, t} \\
& +\frac{4 \alpha^{3}}{3 \sqrt{\pi}} \mu_{i, s}+\delta^{s z} 4 \pi \sum_{j \in \mathcal{D}_{\text {cut }}} \mu_{j, z}
\end{aligned}
$$

until the criterion eq. (25) is verified.

Finally, the energy of the new configuration is computed with eq. (12) and the move is accepted or rejected according to the standard Metropolis algorithm. Note that this process is just a convenient way for solving eq. (5) ie computing, for a given set $\left\{\boldsymbol{r}_{i}\right\}$ of particles coordinates, the distribution of dipole moments. The electrostatic energy depends thus only on $\left\{\boldsymbol{r}_{i}\right\}$, as with ordinary (pair additive) interactions. Since the sampling of the configuration space is done here by single particle translation moves (the first step above from to $\boldsymbol{r}_{i_{\mathrm{mv}}}^{\text {new }}$ to $\boldsymbol{r}_{i_{\mathrm{mv}}}^{\text {old }}$ ), the detailed balance condition is indeed satisfied by the standard acceptation rule. Now, the error in the change in energy after one particle move, due to the approximate treatment of the local field, should of course be minimized by taking a large cutoff, as shown below.

\section{Test of the method}

\subsection{Simulation of asymmetric mixtures with polarizable particles in the Canonical Ensemble}

We first tested this method on a model considered in our previous work [14] : a mixture of hard-spheres (diameter $\sigma_{s}$ ) and polarizable hard-spheres (diameter $\sigma$ ) confined between two parallel walls. The pore width is $H=3 \sigma$, and the size ratio $\sigma / \sigma_{s}=2$. The other parameters are the same as in part $\mathrm{C}$ of [14] but the permanent dipole $\mu^{*}=1.2$ in the external field $E^{*}=20$ (defined for convenience as $E^{*}=E \sqrt{\sigma^{3} / \epsilon_{w}}$ from the actual field strength $\mathrm{E}$ and wall-small spheres interaction strength $\epsilon_{w}$ ) is replaced by an induced one with $\alpha_{p}=0.06 \sigma^{3}$.

For the Ewald summation we took a parameter $\alpha=7 / L$ were $L$ is the lateral box size. The gap width is $\gamma L-H$. As in reference [19], we took $\gamma=10$. For the terms in reciprocal space we took $n^{2}=80$. No significant deviation was found [19] between the exact electrostatic energy for a lattice of permanent dipoles and the one obtained with these values of the Ewald parameters and gap width. We made a similar comparison for a slab of three layers of induced dipoles arranged on a square lattice. For $\alpha_{p}=0.06 \sigma^{3}$ and typical field strengths, we again found no significant deviation between the exact and the Ewald sum, with $\gamma=10$. To estimate the sensitivity to this parameter, we considered the value $\gamma=1$. We found no significant variation of the average quantities, compared to $\gamma=10$. For example, when going from $\gamma=1$ to $\gamma=10$ we found a difference in the average energy per particles $\delta U / U \approx 3.10^{-6}$ for $E^{*}=20$ and the densities considered below. For the slab width 
considered here, $H=3 \sigma$ and $\gamma=1$, the ratio $\gamma L / H$ is indeed already greater than three even for the smallest lateral width $L=10.25 \sigma$. Since optimizing this aspect of the method was not our objective here, we kept the somewhat rather large value $\gamma=10$ for safety. Furthermore, when the dipoles are induced by an external field, there is a significant dipole-field contribution to the variation in electrostatic energy after one particle move. A very accurate treatment of the long-range dipole-dipole contributions seems then less pressing, especially in view of the fact that the largest error comes from the partial update itself, as discussed below.

To test the update method, we performed a converged run of $N_{\text {step }}=40$ Kcycles (one cycle involves $N_{L}+N_{s}$ trial moves ) with a cut-off distance $R_{\text {cut }}=3 \sigma$. The same run was repeated with full update. To begin with, $N_{L}=129$ polarizable particles and $N_{s}=1032$ small hard-spheres were used in both cases. While it takes 3 days on a 4-processors computer to complete the first simulation, the same run with full update takes about 15 days on the same machine.

As the time saving is important, the effect of the approximate treatment for the local field must be discussed. Fig. 2 shows the mean change in the magnitude of the dipole moment for the particles that are separated by a lateral distance greater than $R$ from the displaced particle, for full and partial update with $R_{\text {cut }}=3 \sigma$.

The direct effect of the cut-off distance is to ignore the variation of the dipole moment beyond $R_{\text {cut }}$. The latter is more than 10 times smaller than the maximum value around $R=1$. Inside the cylinder the variations differ slightly, but are on the whole quite consistent.

We now evaluate the impact of the cut-off method on the energy of the system. With the same parameters a relative difference $\left|U_{\text {Full }}-U_{R_{\text {cut }}=3 \sigma}\right| / U_{\text {Full }} \approx 8.10^{-6}$ is found between the two methods, for each configuration. This difference does not increase during the simulation. This suggests that the equilibrium states sampled by the two methods are not too different.

This is confirmed by Fig. 3 which shows the change in energy $\Delta U$ induced by the trial move of one particle, in the two methods, for two box sizes corresponding to the same state: the former box with $N_{L}=129$ and $N_{s}=1032$ and a bigger one having a twice larger surface, with $N_{L}=258$ and $N_{s}=2064$. Fig. 3a and Fig. 3c show that $\Delta U_{R_{\text {cut }=3 \sigma}}$ remains close to $\Delta U_{\text {Full }}$. The rare cases where the latter is zero but not the former show directly the magnitude of the error due to the cut-off, compared to $k_{B} T$. Since the difference between the two quantities is always very small compared to $k_{B} T$, the acceptance probability of the moves should remain nearly the same. Using $\Delta U_{R_{\text {cut }}=3 \sigma}$, instead of the exact one, should thus not introduce a significant bias, as mentioned above. Fig. 3b and Fig. 3d show $\Delta U_{R_{\text {cut }}=3 \sigma}$ versus $\Delta U_{\text {Full }}$. In this example, the difference between the two quantities increases with the magnitude of $\Delta U_{\text {Full }}$. The points corresponding to $\Delta U_{R_{\text {cut }}=3 \sigma}$ seem to be distributed on a line with a slope slightly different from unity and not as a random noise about the line of unit slope. This observation could be used to improve the method by correcting $\Delta U_{R_{\text {cut }}=3 \sigma}$ (say by a linear fit that forces the equality with the exact result). But without this, we find that the cut-off induces a change in the Markov chain after approximately 250 trial moves in the smaller box. We repeated the same tests with $R_{\text {cut }}=\sigma$. Then the Markov chain obviously deviates faster (after about 50 trials). The choice $R_{\text {cut }}=3 \sigma$ seems thus here a good compromise for reducing the computer time without significantly affecting the simulation dynamics.

We finally compare in Fig. 4a the density profiles of the dipoles for the system with $N_{L}=129$ in the two methods. We observe no significant difference due to the cut-off. To investigate the size effect in a converged simulation, we considered the bigger box with $N_{L}=258$ and $N_{s}=2064$. In the cut-off method (with $R_{\text {cut }}=3 \sigma$ ) 
a simulation with 40 Kcycles, takes 9 days on the same 4-processors computer. This gives a scaling time factor of $N^{3 / 2}$ for the algorithm with cut-off. The corresponding density profile of the polarizable hard-spheres is shown in Fig. 4b. No significant size effect is found in comparison with the simulation with 129 dipoles. In the full update method now, we estimated that the same simulation would take about 6 months (on the basis of limited runs of about 100 cycles). Even so, a single check would be insufficient. We thus cannot discuss here the size effect in the method with full update, beyond the data shown as a function of the number of particle moves in Fig. 3a and Fig. 3c.

From a more physical perspective now, we may note that the results are similar to those shown in [14] for permanent dipoles: without the field, the density profile of the large particles is structured at the scale of the small ones. In a normal electric field, this layering is replaced by one at the scale of the large polarizable particles.

Additional tests of the size effects and of different choices of $R_{\text {cut }}$ should of course be performed to assess more quantitatively the consequences of this partial update method (this last question is not specific to the slab geometry considered here). At the present stage, we found it more important to examine if the general idea remains applicable in an open system.

\subsection{Grand-canonical simulation of a non-additive mixture of pure and polarizable hard-spheres}

This model is motivated by our recent study [15] in which we have shown how the composition of a fluid comprising dipolar particles in an open pore may be controlled by applying an electric field. For mixtures with non-additive interactions, a population inversion occurs in the pore beyond a certain threshold value of the applied field: starting from the situation of a pore in equilibrium with a bulk mixture having widely different concentrations of the two components, a jump in the adsorption of the minority component occurs when the field strength is varied. After the threshold, the pore becomes filled with this species, while the former majority component simultaneously desorbs.

In ref. [15] the dipoles were taken permanent. We relax here this constraint for the reasons explained in the introduction (polarizable colloids). We thus consider a mixture of hard-spheres (species (1)) and polarizable hard-spheres (species (2)) with non-additive diameters. For species (2) we took a polarizability $\alpha_{p}=0.04 \sigma^{3}$ (in order to model colloidal particles). A reduced field strength $E^{*}=E\left(\sigma^{3} / k T\right)^{1 / 2}$ will be used. For simplicity, we took $\sigma_{2}=\sigma_{1}=\sigma$. For the non additivity parameter (defined from the cross diameter $\sigma_{i j}=1 / 2\left(\sigma_{i}+\sigma_{j}\right)(1+\delta)$ ) we took $\delta=0.2$. The mixture is confined in a pore of width $H=3 \sigma$. The bulk fluid that fixes the chemical potentials in the pore is the same mixture of non additive hard-spheres, but without polarization, because the electric field is applied only in the pore. The total reservoir density is $\rho_{b}=0.53$. The bulk mole fraction of the polarizable particles is $x_{2}=0.02$. The simulation in the pore is done in the Grand-canonical ensemble. The reduced density in the pore is $\rho=\langle N\rangle \sigma^{3} / V$, with $\langle N\rangle$ the average number of particles and $V=S(H-\sigma)$.

For the translation moves, we first study the effect of the cut-off on the relative mean variation of the dipole moment after the move of one particle. Fig. 5 shows the result for $R_{\text {cut }}=3 \sigma$ and $E^{*}=5$. Although $<\frac{|\Delta \boldsymbol{\mu}|}{|\boldsymbol{\mu}|}>$ is larger than in Fig. 2, due to a different dynamics - the maximum displacement of the particles $(\delta r=0.3 \sigma)$ is 5 times bigger, we again find a negligible effect beyond $R_{\text {cut }}$.

The consequences on the change in energy is shown in Fig. 6 . The same features as in Fig. 3 are observed. The error induced by the cut-off is still very small compared 
to the change in energy after one particle move (with the same remark as with for Fig. 3a when Delta $\left.U_{\text {Full }}=0\right)$. The smaller energies are due to lower values of the field and polarizability. We finally measure that a change in the Markov chain occurs only after 100 trial moves, probably because of the larger accepted displacement.

Similar tests may be done for the deletion or the insertion of the dipoles. To anticipate the effect of larger changes than with translation moves only, we took a larger cut-of, $R_{\text {cut }}=5 \sigma$. The iterations are then much longer : about one month on the same 4-processors machine, for comparable values of the parameter than in the canonical simulation. Needless to say, the simulation with full update remains equally problematic.

In Fig. 7, we present the population inversion in the pore that has been discussed in ref. [15], but now for polarizable particles. In this case, the inversion is still present but it occurs for a field that is 4 times stronger than for permanent dipole [15]. These results depend of course on the value of $\alpha_{p}$ and the comparison with permanent dipole is not immediate, since the dipole moment changes with the applied field. An accurate treatment of the local field will of course be necessary to obtain accurate values (for example for the threshold field). The very question of the occurrence of the population inversion should however not depend on this, since it has been observed even without dipoles, by simply changing then the bulk density.

\section{Conclusion}

In this paper, we presented a method for studying confined polarizable fluids by Monte Carlo simulation that combines a slab-adapted Ewald method with an efficient strategy for computing the induced dipoles at each Monte Carlo step. Since we consider a slab geometry, the long-range interactions are handled by the method of Ewald sums adapted to this geometry rather than the reaction field method. To reduce the amount of computer time required by a full update of the dipole moments, we have proposed a simple update strategy that uses a cut-off distance, in the same spirit as the PAPI methods for bulk systems. We have shown that the partial update allows a large gain in computer time while preserving the consistency of the Markov chains over a significant number of Monte Carlo particle displacements. We suggested how this method could be improved to ensure a more consistent calculation of the energy when necessary. This method opens up the possibility to study confined polarizable fluids by $\mathrm{MC}$ simulation with a reasonable restitution time. As with bulk systems, however, the choice of the cut-off distance must be checked on a representative number of Monte Carlo steps before attempting a fully converged simulation. Other improvements specific to the slab geometry, such as the ELC method for reducing the gap width, should also be considered to reduce even further the length of the simulation.

\section{References}

[1] M. Caillol, D. Levesque, J. J. Weis, P. G. Kusalik and G.N. Patey, Molec. Phys. 55, 65 ( 1985 )

[2] D. Levesque and J. J. Weis, J. Stat. Phys. 4029 (1985)

[3] S. H. Lee, J. C. Rasaiah and J. B. Hubbard, J. Chem. Phys. 85, 5232 (1986).

[4] P. G. Kusalik, Molec. Phys. 6767 (1989)

[5] P. Jedlovszky, and J. Richardi, J. chem. Phys. 110, 8019 (1999).

[6] F. Moučka, M. Rouha and I. Nezbeda, J. Chem. Phys. 126, 224106 (2007).

[7] M. Předota; P. T. Cummings; A. A. Chialvo, Molec. Phys. 99, 349 ( 2001); M. Předota; P. T. Cummings; A. A. Chialvo, Molec. Phys. 100, 2703 (2002)

[8] C. Holm, Efficient Methods for Long Range Interactions in Periodic Geometries Plus One Application, NIC series 23, 195 (2004). 
1

3

4

5

[9] G. Zhu, X Luo, J. Zhang, B. Liu and X Yan, Thin Solid Films 517, 5734 (2009)

[10] J. Zhou, J. Ralston, R. Sedev and D. Beattie, J. Colloid and Interf. Sci. 331,521 (2009); S.P. Stoylov, Colloids and Surfaces B: Biointerfaces 56, 50 (2007)

[11] S. Vaitheeswaran, J. C. Rasaiah, and G.Hummer J. Chem. Phys. 121, 7955 (2004).

[12] D. Bratko, C. D. Daub, K. Leung, and A. Luzar, J. Amer. Chem Soc 127, 2504 (2007).

[13] J. Jordanovic and S. H. L. Klapp, Phys. Rev. Lett. 101, 038302 (2008).

[14] C. Brunet, J. G. Malherbe, and S. Amokrane, J. Chem. Phys. 130, 134908 (2009).

[15] C. Brunet, J. G. Malherbe, and S. Amokrane, J. Chem. Phys. 131, 221103 (2009).

[16] C. J. M. Böttcher, 'Theory of Electric polarization' Elsevier, Amsterdam, (1952).

[17] F.J. Vesely, J. Comput. Phys. 24, 361(1977)

[18] I. -C. Yeh and M. L. Berkowitz, J. Chem. Phys. 111, 3155 (1999).

[19] S. H. L. Klapp and M. Schoen, J. Chem. Phys. 117, 8050 (2002).

[20] A. Arnold, J. de Joannis, and C. Holm , J. Chem. Phys. 117, 2496 (2002) ; J. de Joannis, A. Arnold, and C. Holm, J. Chem. Phys. 117, 2503 (2002).

[21] A. Brodka, Chem. Phys. Lett. 400, 62 (2004).

[22] T. M. Nymand and P. Linse, J. Chem. Phys. 112, 6152 (2000).

[23] J. J. Cerda, V. Ballenegger O. Lenz and C. Holm, J. Chem. Phys. 129234104 (2008) 
12

Figures
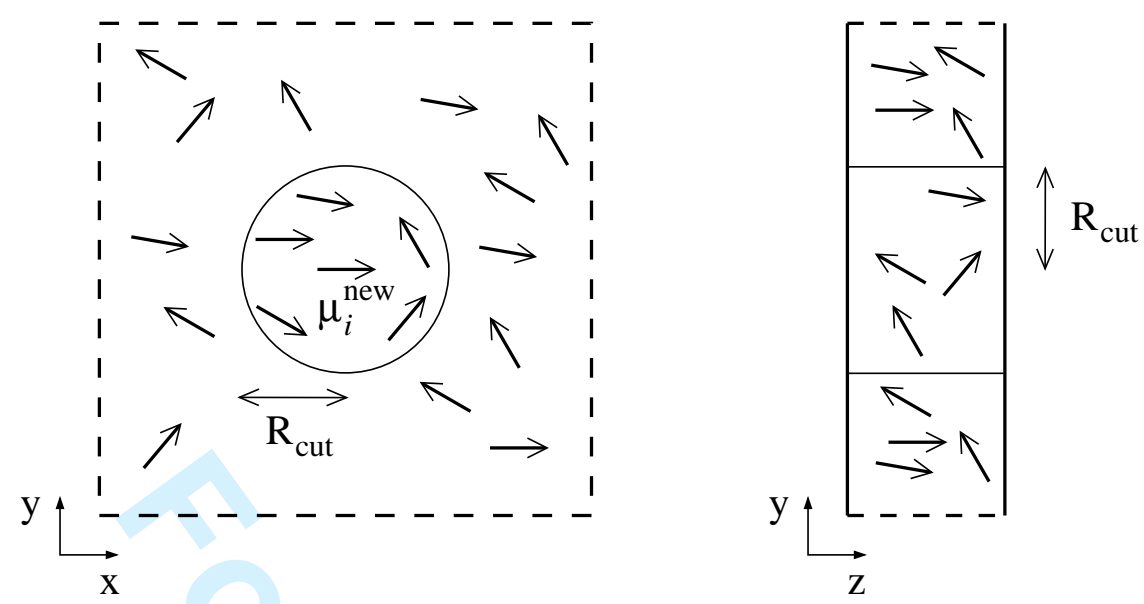

Figure 1.: Schematic view of the cylindrical cut-off in the pore.

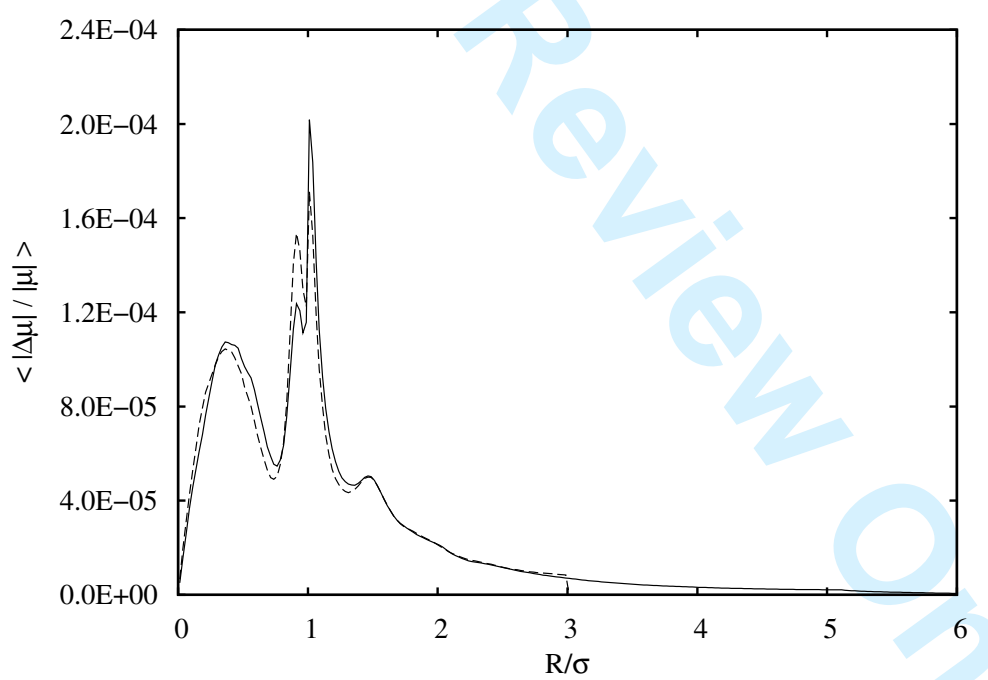

Figure 2.: Relative mean variation $\left\langle\frac{|\Delta \boldsymbol{\mu}|}{|\boldsymbol{\mu}|}\right\rangle$ vs the lateral distance $R=\sqrt{x^{2}+y^{2}}$ from the displaced particle. line: full update process, dashed line: Iteration with $R_{\text {cut }}=3 \sigma$ notice the drop to zero for $r>R_{\text {cut }}$ 
1

3

4

5

6

7

8

9

10

11

12

13

14

15

16

17

18

19

20

21

22

23

24

25

26

27

28

29

30

31

32

33

34

35

36

37

38

39

40

41

42

43

44

45

46

47

48

49

50

51

52

53

54

55

56

57

58

59

60

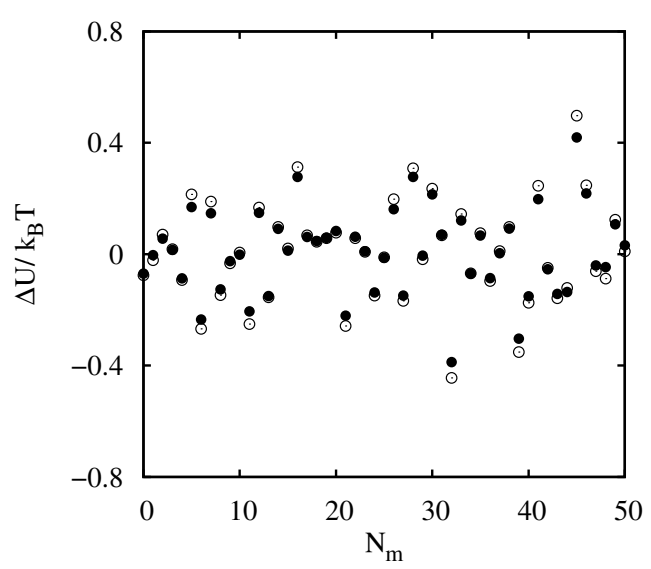

(a) Change in energy $\Delta U / k_{B} T$ after one particle move versus number of particle moves $N_{m}$. Empty circles:

$R_{\text {cut }}=3 \sigma ;$ filled circles: full iterations

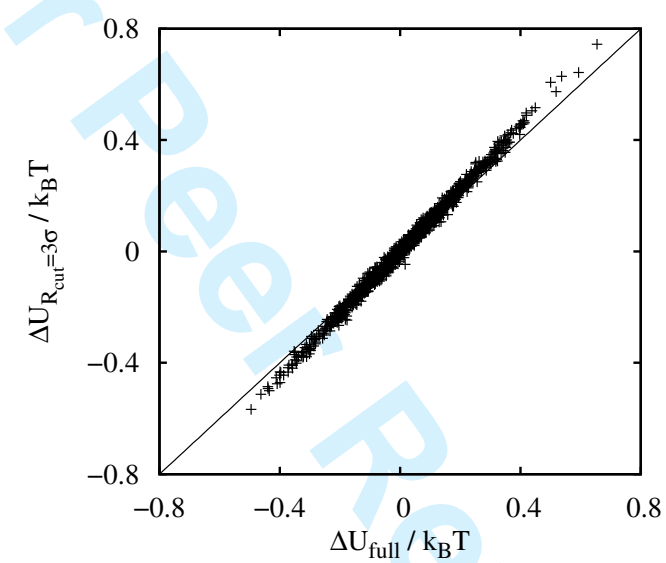

(b) Change in energy with cutoff $\Delta U_{R_{\text {cut }}=3 \sigma} / k_{B} T$ vs full change $\Delta U_{\mathrm{Full}} / k_{B} T$ : crosses. The line with unit slope would correspond to $\Delta U_{R_{\text {cut }}=3 \sigma} / k_{B} T=$ $\Delta U_{\mathrm{Full}} / k_{B} T$

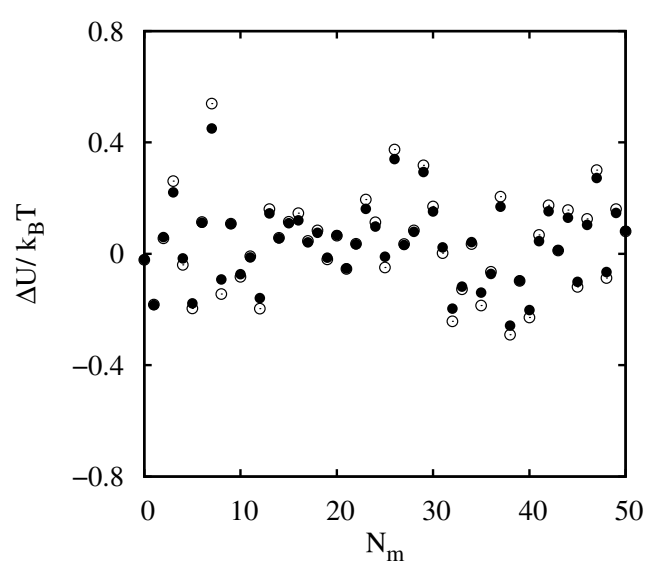

(c) Same caption as in Fig. 3a

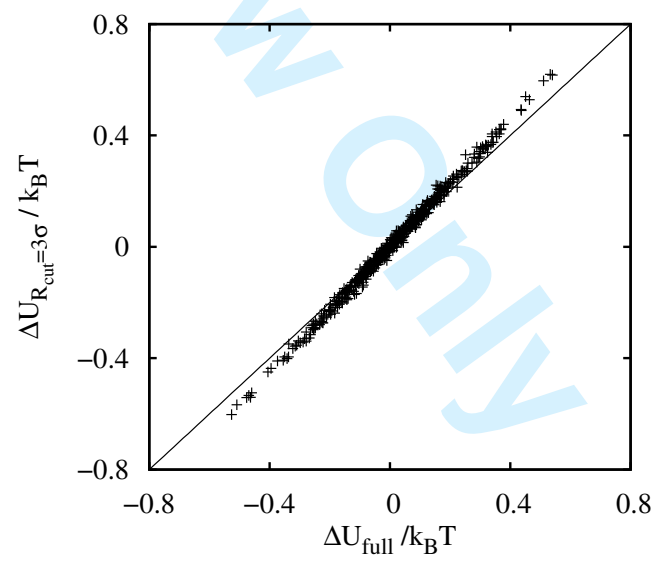

(d) Same caption as in Fig. 3b

Figure 3.: Effect of the cut-off distance on the energy change $\Delta U / k_{B} T$ induced by the trial moves.
(a) and (b): $N_{L}=129$ and $N_{s}=1032$;
(c) and (d)
(d) $N_{L}=258$ and $N_{s}=2064$ 


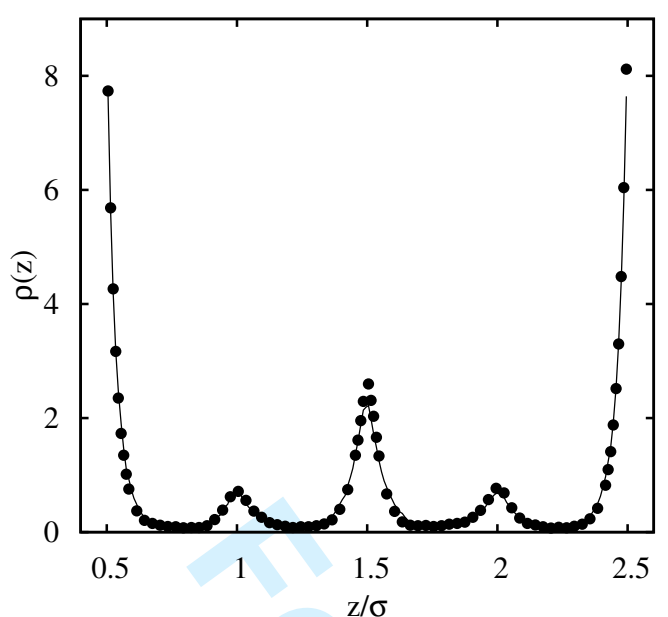

(a) Simulation with $N_{L}=129, E^{*}=20$, Filled circles: $R_{\mathrm{cut}}=3 \sigma$, line: Full update

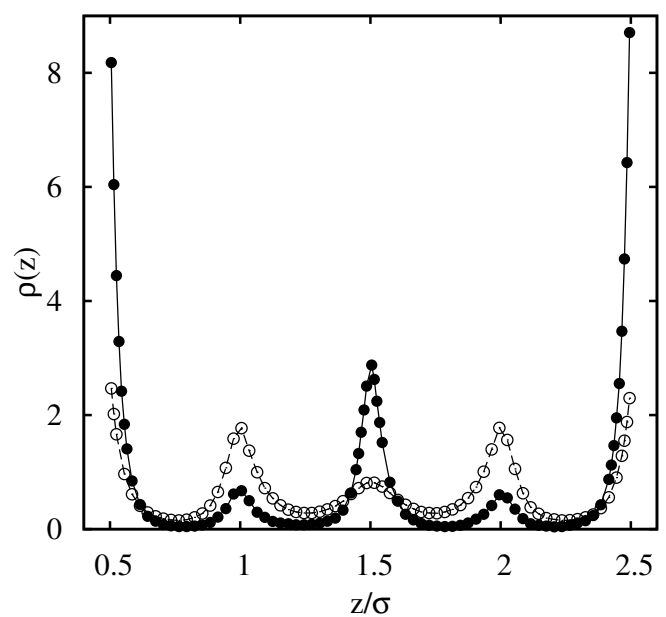

(b) Simulation box with $N_{L}=258$

Open circles: $E^{*}=0$, Filled circles: $E^{*}=20$. Lines are a guide to the eye

Figure 4.: Density profiles of polarizable particles in an external normal electric field.

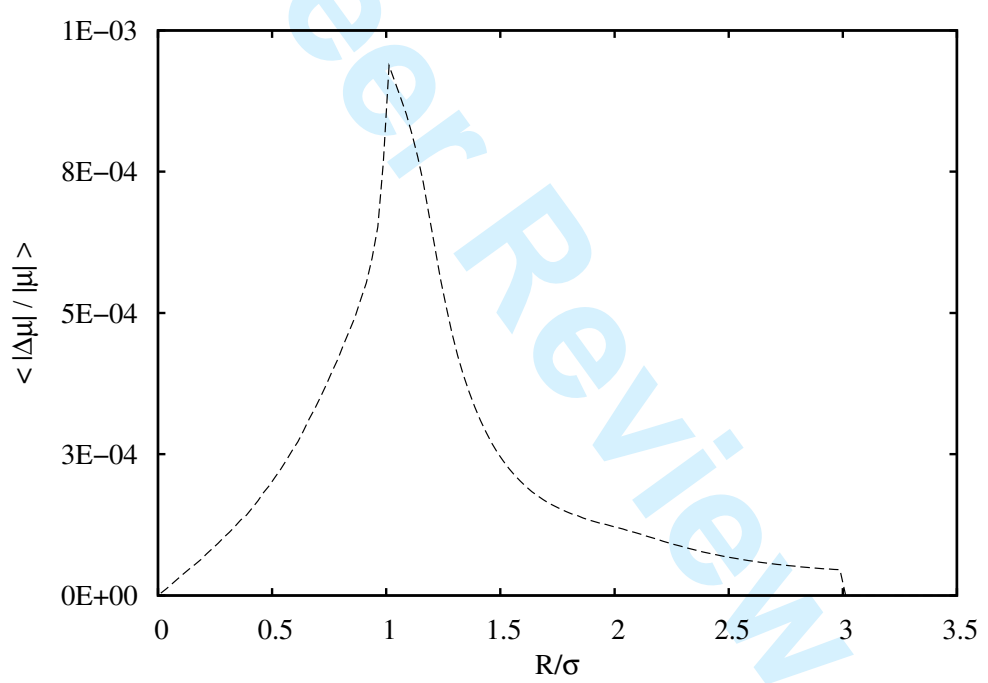

Figure 5.: Relative mean variation $\left\langle\frac{|\Delta \boldsymbol{\mu}|}{|\boldsymbol{\mu}|}>\right.$ vs the lateral distance $R=\sqrt{x^{2}+y^{2}}$ from the displaced particle. dotted line : Iteration with $R_{\text {cut }}=3 \sigma$ 
1

3

4

5

6

7

8

10

11

12

13

14

15

16

17

18

19

20

21

22

23

24

25

26

27

28

29

30

31

32

33

34

35

36

37

38

39

40

41

42

43

44

45

46

47

48

49

50

51

52

53

54

55

56

57

58

59

60

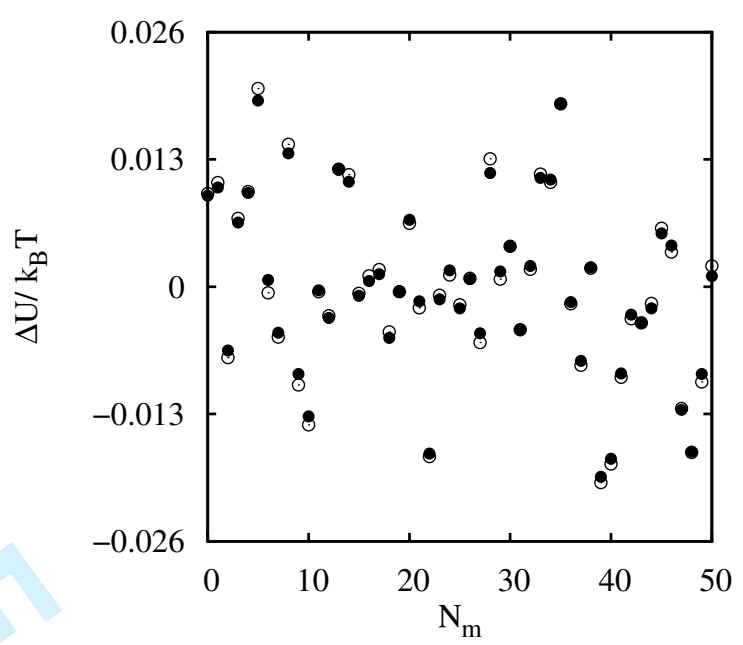

(a) Change in energy $\Delta U / k_{B} T$ after one particle move versus number of particle moves $N_{m}$. Empty circles: $R_{\mathrm{cut}}=3 \sigma ;$ filled circles: full iterations

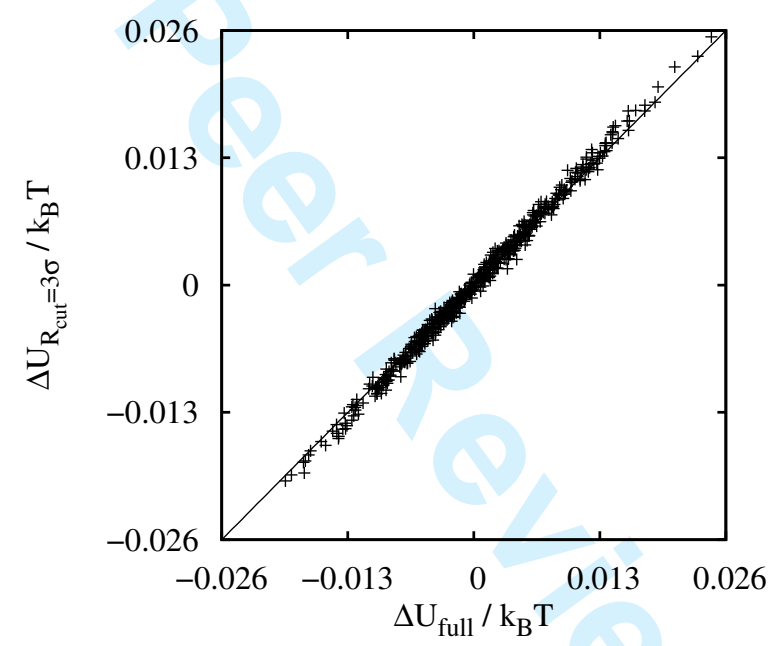

(b) Change in energy with cutoff $\Delta U_{R_{\text {cut }}=3 \sigma} / k_{B} T$ vs full
change $\Delta U_{\text {Full }} / k_{B} T$ : crosses. The line with unit slope would
correspond to $\Delta U_{R_{\text {cut }}=3 \sigma} / k_{B} T=\Delta U_{\text {Full }} / k_{B} T$

Figure 6.: Effect of the cut-off distance on the energy change $\Delta U / k_{B} T$ induced by the trial moves. 


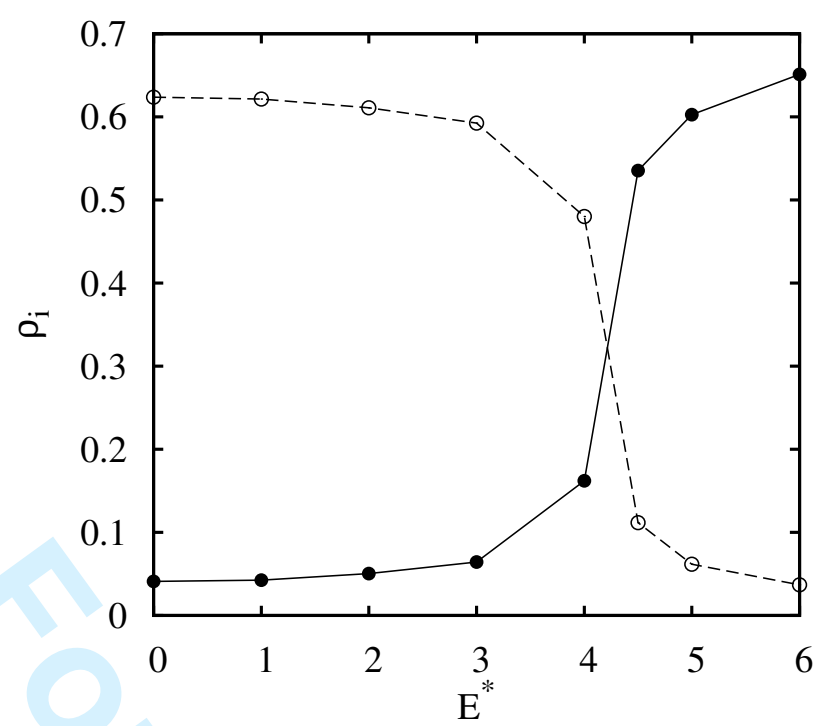

Figure 7.: Electric field controlled population inversion for a non-additive mixture of hard-spheres and polarizable hard-spheres confined in a slit pore. The total reservoir density is $\rho_{b}=0.53$. The bulk mole fraction of the polarizable hardspheres is $x_{2}=0.02 . \rho_{i}$ : density of each species; $E^{*}$ : applied field strength Filled circles: polarizable hard-spheres, Open circles: hard-spheres. Lines are a guide to the eye. 